\title{
A PARTICLE FINITE ELEMENT METHOD FOR MACHINING SIMULATIONS
}

\author{
Rodríguez J.M. ${ }^{1}$, Jonsén P. ${ }^{2}$, and Svoboda A. ${ }^{2}$ \\ ${ }^{1}$ Division of Mechanics of Solid Materials \\ Department of Engineering Sciences and Mathematics. Lulea University of Technology \\ e-mail: rodjua@ltu.se \\ ${ }^{2}$ Division of Mechanics of Solid Materials \\ Department of Engineering Sciences and Mathematics. Lulea University of Technology \\ e-mail: par.jonsen@1tu.se and ales.svoboda@ltu.se
}

Keywords: Updated Lagrangian formulation - Particle Finite element method - Metal cutting - Mixed Stabilized method.

\begin{abstract}
Metal cutting process is a nonlinear dynamic problem that includes geometrical, material, and contact nonlinearities. In this work a Lagrangian finite element approach for simulation of metal cutting processes is presented, based on the so-called Particle Finite Element Method (PFEM). The governing equations for the deformable bodies are discretized with the FEM via a mixed formulation using simplicial elements with equal linear interpolation for displacements, pressure and temperature. The use of PFEM for modeling of metal cutting processes includes the use of a remeshing process, $\alpha$-shape concepts for detecting domain boundaries, contact mechanics laws and material constitutive models. The merits of the formulation are demonstrated in the solution of $2 D$ and $3 D$ thermally-coupled metal cutting processes using the particle finite element method. The method shows good results and is a promising method for future simulations of thermally/coupled machining processes.
\end{abstract}




\section{INTRODUCTION}

Numerical simulation of machining had a tremendous development in the last twenty years [1-5]. Thanks to the increasing computer power, it can provide excellent results with reasonable computational times. Thanks to its flexibility and robustness commercial FEM codes are standard tools in the industry.

However, the main limitation of the FEM in this kind of application is that the quality of the results depends on the mesh. In the problems characterized by large deformations, such as the machining processes, if a Lagrangian formulation is used, the mesh moves with material and the elements become so distorted that numerical results lose their validity, unless a remeshingrezoning technique is used[1,2]. This improves robustness but, at the same time, the computational time increases and supplementary computational errors are introduced, in the form of numerical, artificial, diffusion of the results.

Metal cutting has been simulated also with Eulerian formulations [6] and arbitrary Lagrangian-Eulerian (ALE) [7-9], but for all cases additional drawbacks appear.

A possible alternative is the use of meshless methods. Actually, different mesh-free techniques have been developed in the last years $[5,10,11]$ but in many cases the improvement of the results quality, with the same number of degrees of freedom, is vanished by the very high computational time of shape functions.

In this paper an innovative approach named Particle Finite Element Method (PFEM) has been used. PFEM overcomes the main difficulties in the numerical simulation of metal cutting, specifically, large strains and angular distortions, multiple contacts and self-contact, generation of new boundaries and fracture with multiple cracks and defragmentation.

The outline of this work is as follows. In the following section, the solid-dynamics equations in a Lagrangian framework are introduced, a space-time finite element semi-discretization is presented and the stabilization of the incompressibility condition is described. In section 3 the basic ideas of the PFEM are illustrated. In Section 4 the constitutive model used is explained, and finally a variety of machining and cutting problems are solved in Section 5 to show the efficiency of the proposed method.

\section{GOVERNING EQUATIONS}

Let $\mathbf{x}$ denote the current position of a solid particle in the current configuration at time $t$, $\mathbf{u}=\mathbf{u}(\mathbf{x}, t)$ its displacement, $\theta=\theta(\mathbf{x}, t)$ its temperature, $p=p(\mathbf{x}, t)$ its pressure and $\sigma=\sigma(\mathbf{x}, t)$ the Cauchy stress tensor at time $t \in[0, T]$. For a solid, momentum, mass conservation and energy write as

$$
\begin{aligned}
& \nabla \cdot \sigma+\rho \mathbf{b}=0 \quad \text { in } \Omega \\
& \frac{1}{\kappa} p-\ln (J)=0 \text { in } \Omega \\
& c \frac{D \theta}{D t}-\nabla \cdot(k \nabla \theta)+Q=0 \quad \text { in } \Omega
\end{aligned}
$$

where $\Omega$ is the volume occupied by the solid in the current configuration, $\rho$ is the density, $c$ the specific heat, $k$ the thermal conductivity, $J$ is the determinant of the deformation gradient and $\mathbf{b}$ the external body forces. The initial conditions are prescribed assigning the initial displacement $\mathbf{u}_{0}(\mathbf{x})$ and initial velocity $\mathbf{v}_{0}(\mathbf{x})$ at $t=0$ whereas both Dirichlet and Neumann type boundary conditions are imposed as: 


$$
\begin{aligned}
& \mathbf{u}(\mathbf{x}, t)=\overline{\mathbf{u}}(\mathbf{x}, t) \quad \forall \mathbf{x} \in \Gamma_{D} \\
& \mathbf{v}(\mathbf{x}, t)=\overline{\mathbf{v}}(\mathbf{x}, t) \quad \forall \mathbf{x} \in \Gamma_{D} \\
& \theta(\mathbf{x}, t)=\overline{\mathbf{T}}(\mathbf{x}, t) \quad \forall \mathbf{x} \in \Gamma_{D \theta} \\
& \sigma(\mathbf{x}, t) \cdot \mathbf{n}=\overline{\mathbf{h}}(\mathbf{x}, t) \quad \forall \mathbf{x} \in \Gamma_{N} \\
& -k \nabla \theta \cdot \mathbf{n}=\overline{\mathbf{q}}(\mathbf{x}, t) \quad \forall \mathbf{x} \in \Gamma_{N \theta}
\end{aligned}
$$

where $\overline{\mathbf{u}}(\mathbf{x}, t), \overline{\mathbf{v}}(\mathbf{x}, t), \overline{\mathbf{T}}(\mathbf{x}, t), \overline{\mathbf{h}}(\mathbf{x}, t)$ and $\overline{\mathbf{q}}(\mathbf{x}, t)$ are assigned functions, $\mathbf{n}$ denotes the outward normal to the boundary and $\Gamma_{D} \cup \Gamma_{N}=\partial \Omega$ and $\Gamma_{D T} \cup \Gamma_{N T}=\partial \Omega$

\subsection{Space discretization}

Introducing a Galerkin isoparametric finite element discretization, the semi-discrete form of equations (1)

$$
\begin{aligned}
& \mathbf{F}_{\mathbf{u}, \text { int }}(\mathbf{u}, p)-\mathbf{F}_{e x t}=0 \\
& \mathbf{F}_{\mathbf{p}, \text { press }}(p)-\mathbf{F}_{p, v o l}(\mathbf{u})+\mathbf{F}_{p, s t a b}(p)=0 \\
& \mathbf{F}_{\theta, \text { dyn }}(\dot{\theta})-\mathbf{F}_{\theta, \text { int }}(\theta)+\mathbf{F}_{\theta, e x t}(\theta)=0
\end{aligned}
$$

Where

$$
\begin{aligned}
& \mathbf{F}_{\mathbf{u}, \text { int }}(\mathbf{u}, p)=\int_{V} \mathbf{B}_{u}^{T} \sigma d V \\
& \mathbf{F}_{\mathbf{u}, e x t}=\int_{V} \mathbf{N}^{T} \mathbf{b} d V-\int_{\Gamma_{N}} \mathbf{N}^{T} \overline{\mathbf{h}} d \Gamma \\
& \mathbf{F}_{p, p r e s s}(p)=\int_{V} \frac{1}{\kappa} \mathbf{N N}^{T} p d V \\
& \mathbf{F}_{p, v o l}(\mathbf{u})=\int_{V}^{V} \mathbf{N}^{T} \ln (J) d V \\
& \mathbf{F}_{p, s t a b}(p)=\int_{V^{(e)}} \frac{\alpha}{\mu} p^{(e)} \mathbf{N}^{(e)} \mathbf{N}^{(e) T}-\tilde{\mathbf{N}}^{(e)} \tilde{\mathbf{N}}^{(e) T} d V \\
& \mathbf{F}_{\theta, \text { int }}(\theta)=\int_{V}^{V^{(e)}} \mathbf{B}_{\theta}^{T} \mathbf{q} d V-\int_{V} \mathbf{N}^{T} D_{\text {int }} d V \\
& \mathbf{F}_{\theta, e x t}=\int_{\Gamma_{N T}}^{V} \mathbf{N}^{T}(\mathbf{q . n}) d \Gamma \\
& \mathbf{F}_{\theta, d y n}(\dot{\theta})=\int_{V} c \mathbf{N N}^{T} \dot{\theta} d V
\end{aligned}
$$

In the above expressions, $\mathbf{N}$ are the shape functions, $\tilde{\mathbf{N}}^{(e)}$ the constant shape function through the element, $\mathbf{B}_{\mathbf{u}}$ and $\mathbf{B}_{\theta}$ are the strain displacement matrix and the gradient-temperature matrix, respectively. Those matrices contain the derivatives of the shape functions used in the interpolation of the problem variables.

In finite element computations, the above force vectors are obtained as the assemblies of element vectors. Given a nodal point, each component of the global force associated with a particular global node is obtained as the sum of the corresponding contributions from the element force vectors of all elements that share the node. In this work, the element force vectors are evaluated using Gaussian quadrature. 


\subsection{Pressure stabilization}

The selected discretization (linear elements for displacement and pressure) is known to lead to spurious oscillations in the pressure field and a stabilization procedure is required. For the present purpose the stabilized formulation called the Polynomial Pressure Projection (PPP) presented and applied to the Stokes equations in [12] is used. The PPP introduces a pressure term in the mass conservation equation $\mathbf{F}_{p, s t a b}$ shown in Eq. (3) and (4). Unlike other stabilization methods, the Polynomial pressure projection (PPP) does not require specification of a mesh dependent stabilization parameter or calculation of higher-order derivatives. The PPP uses a projection on a discontinuous space and as a consequence can be implemented in an elementary level surpassing the need of mesh dependent and problem dependent parameters.

\subsection{Time integration}

A staggered approach[13] is adopted for the purpose of coupling the mechanical and thermal equations. Mechanical and thermal computations are staggered assuming constant temperature during the mechanical step and constant heat generation during the thermal step. A mechanical step is taken first on the current distribution of temperatures and the heat generated is computed as

$$
D_{\text {int }}=\chi \sigma_{y} \dot{\xi}
$$

Where $\chi$ is the Taylor-Quinney coefficient, $\sigma_{y}$ the yield stress and $\dot{\xi}$ the equivalent strain rate. A Newton-Raphson algorithm solves the momentum equation and mass conservation for nodal displacement and pressures. The heat computed in the mechanical problem is transferred to the thermal problem and the balance of energy is discretized using an implicit BackwardEuler algorithm. The solution of discretized energy equation is carried out using a NewtonRaphson algorithm.

\section{DESCRIPTION OF THE METHOD}

The PFEM is a set of numerical strategies combined for the solution of large deformation problems. The PFEM, in the form implemented in the present work, consists of the following conceptual steps

1. Definition of the domain(s) in the last converged configuration, $t={ }^{n} t$, keeping existing spatial discretization.

2. Transference of variables by a smoothing process - from Gauss points to nodes.

3. Discretization of the given domain(s) in a set of particles of infinitesimal size-elimination of existing connectivity.

4. Reconstruction of the mesh through a triangulation of the domain's convex-hull and the definition of the boundary applying the $\alpha$-shape method [14], defining a new spatial discretization .

5. A contact method to recognize the multibody interaction.

6. Transference of information, interpolating nodal variables into the Gauss points.

7. Solution of the system of equations for ${ }^{n+1} t={ }^{n} t+\Delta t$.

8. Go back to step 1 and repeat the solution process for the next time step.

\subsection{Mesh generation and identification of external boundaries}

In a Lagrangian framework the external boundary and the reference volume are defined by the position of the material particles. Every time the mesh is regenerated, the particles belonging 
to the boundary may change and the new boundary nodes (and therefore the particles) have to be identified. The Delaunay triangulation generates the convex hull of the set of particles. Moreover, the convex hull may not be conformal with the external boundaries. A possibility to overcome this problem is to correct the generated mesh using the so-called $\boldsymbol{\alpha}$-shape method[14] to remove the unnecessary triangles from the mesh using a criterion based on the mesh distortion. The $\boldsymbol{\alpha}$-shape method can also be used for the identification of the solid particles which separate from the rest of the domain.

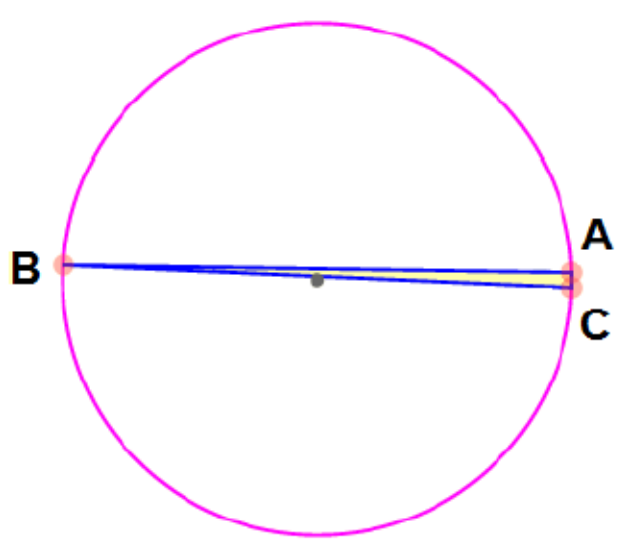

(a) Sharp triangle

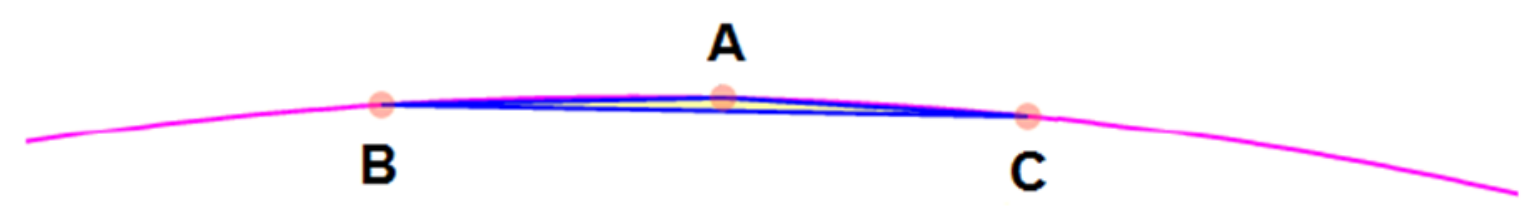

(b)Flat triangle

Figure 1. Triangles with the same mean size and their circumcircles.

In the numerical simulation of metal cutting process, despite the continuous Delaunay triangulation, elements arise with unacceptable aspect ratios. Neither the Alpha Shape method nor the Delaunay triangulation can prevent from the creation of highly distorted elements. Let consider, for example, the triangle depicted in Figure 1(a). The circumradius associated to this type of triangle is not excessively large. The Alpha Shape method and the Delaunay triangulation can only ensure the best physical mesh (so the best discretization that respects the physical boundaries of the domain) for a given cloud of points. However, during the motion, the nodes distribution may be such that it is impossible to avoid the presence of poor quality elements. In order to avoid this eventuality the remeshing with the Delaunay tessellation and the Alpha Shape method must be combined with some additional controls.

For example, for avoiding the presence of sharp elements in the triangulation, as the one 
shown in Figure 1(b), one should monitor the edge lengths of each triangle. Whenever the distance between two nodes is smaller than a prearranged critical length, one of those nodes is removed.

Another type of critical elements are the slivers, so the simplices with null area or volume. These may form when there are three points contained in the same line, or four points laying in the same 3D plane as shown in Figure 2.

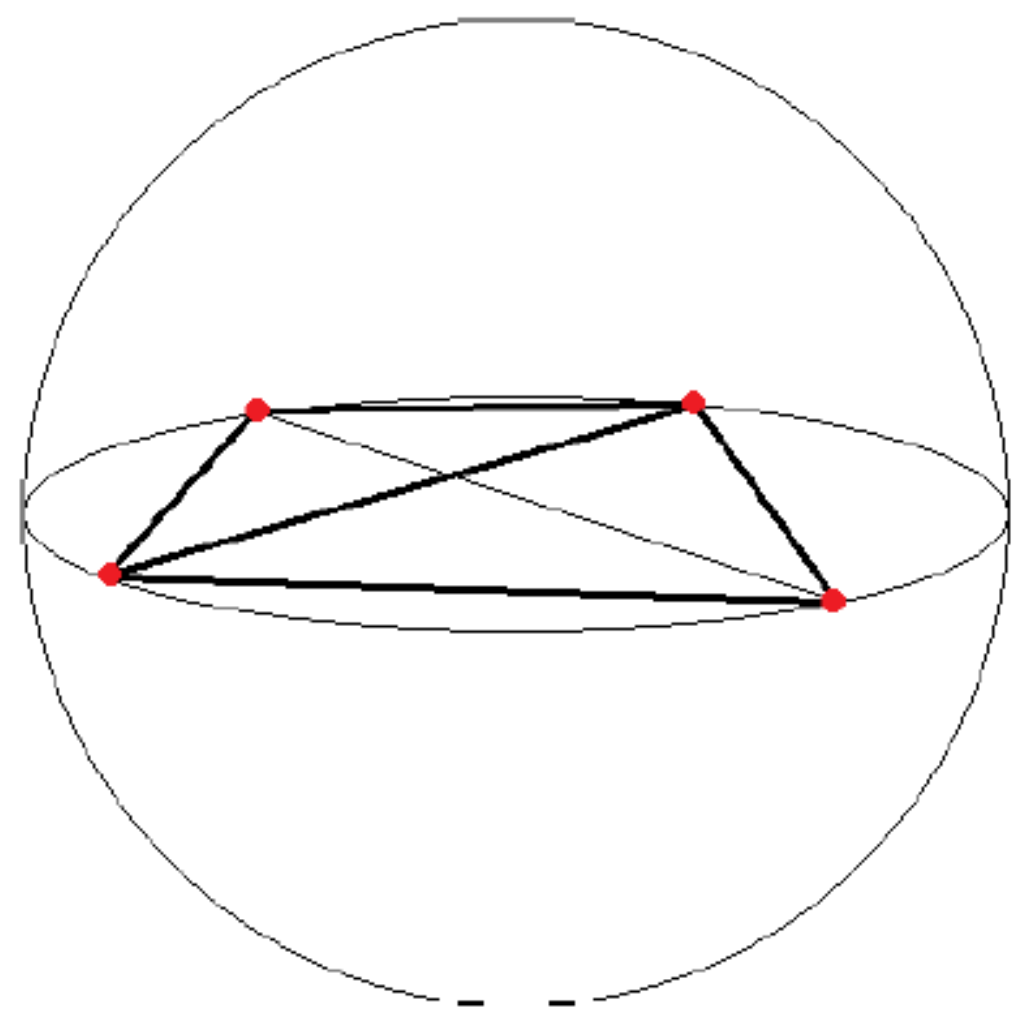

Figure 2. Sliver element in 3D.

In theory, the Alpha Shape method should not allow the formation of those elements because the associated circumradius is huge, see Figure 1(b). However, these elements are critical from the numerical point of view and they can escape to the Alpha Shape control. For all these reasons, the formation of sliver elements must be prevented. In this work, the formation of slivers is prevented by checking the element areas. Whenever the element surface or volume is less than a threshold value, computed for example with respect to the mean area of the mesh, one of the nodes of those elements is removed. Note that this control is also beneficial versus the formation of sharp elements.

After these operations over the nodes, the discretization needs to be updated. It is done in this work modifying locally the mesh and the Alpha Shape control. Once the updated mesh is created, the nodal variables of the new particles are assigned, via interpolation, with the values of the neighbor nodes.

\subsection{Mesh refinement}

The addition of particles is the principal tool, which we employ for sidestepping the difficulties, associated with the deformation-induced element distortion, and for resolving the different scales of the solution. The insertion of nodes is based on the equidistributiom of plastic 
power, such that elements exceeding a prescribed tolerance TOL are targeted for refinement $[13,15]$.

\section{THE CONSTITUTIVE MODEL}

A summary of the $\mathbf{J}_{2}$-plastic flow model with isotropic hardening is shown in Table 1. Table 2 shows the main steps involved in the radial return mapping algorithm and Table 3 shows the expression of the consistent tangent elastoplastic moduli considering non-linear isotropic hardening law known as Johnson-Cook.[16-19]

(i) Multiplicative decomposition of the deformation gradient

\section{$\mathbf{F}=\mathbf{F}^{e} \mathbf{F}^{p}$}

(ii) Free energy

$$
\begin{aligned}
& \psi\left(J, \overline{\mathbf{b}}^{e}, \xi\right):=U(J)+\bar{W}\left(\overline{\mathbf{b}}^{e}\right)+\mathrm{H}(\xi) \\
& U(j)=\frac{1}{2} \kappa \log ^{2}(J), \quad J=\operatorname{det}[\mathbf{F}]=\operatorname{det}\left[\mathbf{F}^{e}\right] \\
& \bar{W}\left(\overline{\mathbf{b}}^{e}\right)=\frac{1}{2} \mu\left(\operatorname{tr}\left[\overline{\mathbf{b}}^{e}\right]-3\right), \overline{\mathbf{b}}^{e}=J^{-\frac{2}{3}} \mathbf{b}^{e}, \mathbf{b}^{e}=\mathbf{F}^{e} \mathbf{F}^{e T}
\end{aligned}
$$

(iii) Mixed Hyperelastic and Hardening response

$\tau(\mathbf{u}, p)=p \mathbf{1}+\mathbf{s}(\mathbf{u}), p=J U^{\prime}(J), \mathbf{s}(\mathbf{u})=\mu \operatorname{dev}\left(\overline{\mathbf{b}}^{e}\right)$

$$
\sigma_{y}=\left(A+B \xi^{n}\right)\left(1+\log \left(\frac{\dot{\xi}}{\dot{\xi}_{\text {ref }}}\right)\right)\left(1-\left(\frac{T-T_{o}}{T_{\text {melt }}-T_{o}}\right)^{m}\right)
$$

(iv) Von-Mises yield condition

$$
\phi(\mathbf{s}, \xi):=\|\mathbf{s}\|-\sqrt{\frac{2}{3}} \sigma_{y}(\xi) \leq 0
$$

(v) Associative flow rule

$$
L_{v} \overline{\mathbf{b}}^{e}=\gamma \operatorname{tr}\left[\overline{\mathbf{b}}^{e}\right] \mathbf{n}, \dot{\xi}=\gamma \sqrt{\frac{2}{3}}, \mathbf{n}=\frac{\mathbf{s}}{\|\mathbf{s}\|}
$$

(vi) Kuhn-Tucker loading/unloading conditions

$\gamma \geq 0, \quad \phi \leq 0, \quad \gamma \phi=0$

(vii) Consistency condition

$\gamma \dot{\phi}=0$ if $\phi=0$

\section{Table 1. Hyperelastic $J_{2}$-flow model at finite strain}

Step 1: Database and initial data $\mu, \mathbf{F}_{n+1}, \overline{\mathbf{b}}_{n}^{e}, \xi_{n}, p_{n+1}$

Step 2: Compute the elastic predictor

$$
\begin{aligned}
& \overline{\mathbf{b}}_{n+1}^{e, t r i a l}=\overline{\mathbf{F}}_{n+1} \overline{\mathbf{b}}_{n}^{e} \overline{\mathbf{F}}_{n+1}^{T}, \overline{\mathbf{F}}_{n+1}=\operatorname{det}\left(\overline{\mathbf{F}}_{n+1}\right)^{-\frac{1}{3}} \overline{\mathbf{F}}_{n+1} \\
& \xi_{n}^{\text {trial }}=\xi_{n} \\
& \mathbf{s}_{n+1}^{\text {trial }}=\mu \operatorname{dev}\left[\overline{\mathbf{b}}_{n+1}^{e, t r i a l}\right], \sigma_{y}^{\text {trial }}=\sigma_{y}\left(\xi_{n}^{\text {trial }}\right) \\
& f_{n+1}^{\text {trial }}=\left\|\mathbf{s}_{n+1}^{\text {trial }}\right\|-\sqrt{\frac{2}{3}} \sigma_{y}^{\text {trial }}
\end{aligned}
$$

Step 3: Check for plastic loading 
IF $: f_{n+1}^{\text {trial }} \leq 0$ THEN

Set $(.)_{n+1}=(.)_{+1}^{\text {trial }}$ and EXIT

END IF

Step 4: Radial return mapping

Set: $\bar{\mu}_{n+1}=\mu \bar{I}_{n+1}^{e}, \bar{I}_{n+1}^{e}=\frac{1}{3} \operatorname{tr}\left[\overline{\mathbf{b}}_{n+1}^{e, t r i a l}\right]$

Solve $\left\|\mathbf{s}_{n+1}^{\text {trial }}\right\|-2 \bar{\mu}_{n+1} \gamma_{n+1}-\sqrt{\frac{2}{3}} \sigma_{y}=0$ for $\gamma_{n+1}$

$\mathbf{n}_{n+1}:=\frac{\mathbf{s}_{n+1}^{\text {trial }}}{\left\|\mathbf{s}_{n+1}^{\text {trial }}\right\|}$

$\mathbf{s}_{n+1}=\mathbf{s}_{n+1}^{\text {trial }}-2 \bar{\mu}_{n+1} \gamma_{n+1} \mathbf{n}_{n+1}, \xi_{n+1}=\xi_{n+1}^{\text {trial }}+\sqrt{\frac{2}{3}} \gamma_{n+1}$

Step 5: Addition of the elastic pressure

$\tau_{n+1}=p_{n+1} \mathbf{1}+\mathbf{s}_{n+1}$

Table 2. Hyperelastic $\mathbf{J}_{2}$-flow model at finite deformations: radial return mapping algorithm

$$
\begin{aligned}
& \mathbf{c}_{n+1}^{d e v}=\left(1-\beta_{1}\right) \mathbf{c}_{n+1}^{\text {dev,trial }}-2 \bar{\mu}_{n+1}\left(\beta_{3} \mathbf{n}_{n+1} \otimes \mathbf{n}_{n+1}+\beta_{4}\left(\mathbf{n}_{n+1} \otimes \operatorname{dev}\left[\mathbf{n}_{n+1}^{2}\right]\right)\right) \\
& \mathbf{c}_{n+1}^{d e v}=2 \bar{\mu}_{n+1}\left(\underline{\mathbf{1}}-\frac{1}{3} \mathbf{1} \otimes \mathbf{1}\right)-\frac{2}{3}\left\|\mathbf{s}_{n+1}^{\text {trial }}\right\|\left(\mathbf{n}_{n+1} \otimes \mathbf{1}_{n+1}+\mathbf{1}_{n+1} \otimes \mathbf{n}_{n+1}\right) \\
& \beta_{0}=1+\frac{\partial \sigma_{y} / \partial \xi_{n+1}}{3 \bar{\mu}}, \beta_{1}=2 \bar{\mu}_{n+1} \frac{\gamma_{n+1}}{\left\|\mathbf{s}_{n+1}^{\text {trial }}\right\|}, \beta_{2}=\left(1-\frac{1}{\beta_{0}}\right) \frac{2}{3} \frac{\left\|\mathbf{s}_{n+1}^{\text {trial }}\right\|}{\bar{\mu}} \gamma_{n+1} \\
& \beta_{3}=1 / \beta_{0}-\beta_{1}+\beta_{2}, \beta_{4}=\left(1 / \beta_{0}-\beta_{1}\right) \frac{\left\|\mathbf{s}_{n+1}^{\text {trial }}\right\|}{\bar{\mu}}
\end{aligned}
$$

Table 3. Hyperelastic $\mathbf{J}_{2}$-flow model at finite deformations: deviatoric consistent elastoplastic moduli

In Table $1 \psi\left(J, \overline{\mathbf{b}}^{e}, \xi\right)$ represents the uncoupled volumetric/isochoric stored-energy function, $U(J)$ the volumetric part of the stored energy function, $\bar{W}\left(\overline{\mathbf{b}}^{e}\right)$ the isochoric part of the energy function defined in terms of the elastic left Cauchy-Green tensor $\overline{\mathbf{b}}^{e}, \tau(\mathbf{u}, p)$ the Kirchhoff stress tensor, $p$ the Kirchhoff pressure, $\mathbf{s}(\mathbf{u})$ the deviatoric component of the Kirchhoff stress tensor. In Table 2 and Table $3(.)_{n}$ and $(.)_{n+1}$ denote the time discrete values of the variable $($. at times $t_{n}$ and $t_{n+1}$, respectively.

\section{APPLICATIONS/NUMERICAL EXAMPLES}

The objective of this section is to show the ability of the PFEM to simulate metal cutting problems in both 2D and 3D, where very large strains (around 10) are present.

\subsection{Orthogonal cutting test: 2D approach}

Our first case is an industrial application consisting of a rigid tool that is cutting a rectangular block of steel. The block has a length of $3.7 \mathrm{~mm}$, a width of $1.8 \mathrm{~mm}$ and a depth of $1 \mathrm{~mm}$. The 
cutting has an imposed velocity of $3333.3 \mathrm{~mm} / \mathrm{s}$, a cutting depth of $0.60 \mathrm{~mm}$, a rake angle of $0^{\circ}$, a clearance angle of $0^{\circ}$ and a tool radius of $0.4 \mathrm{~mm}$. The rigid tool is composed by to straight lines connected by a circular arch on the tool tip with the characteristics of the cutting parameters described, see Figure 3. The workpiece material behavior is given by a Johnson-Cook constitutive law that takes into account strain hardening, strain rate hardening and thermal softening Table 4.

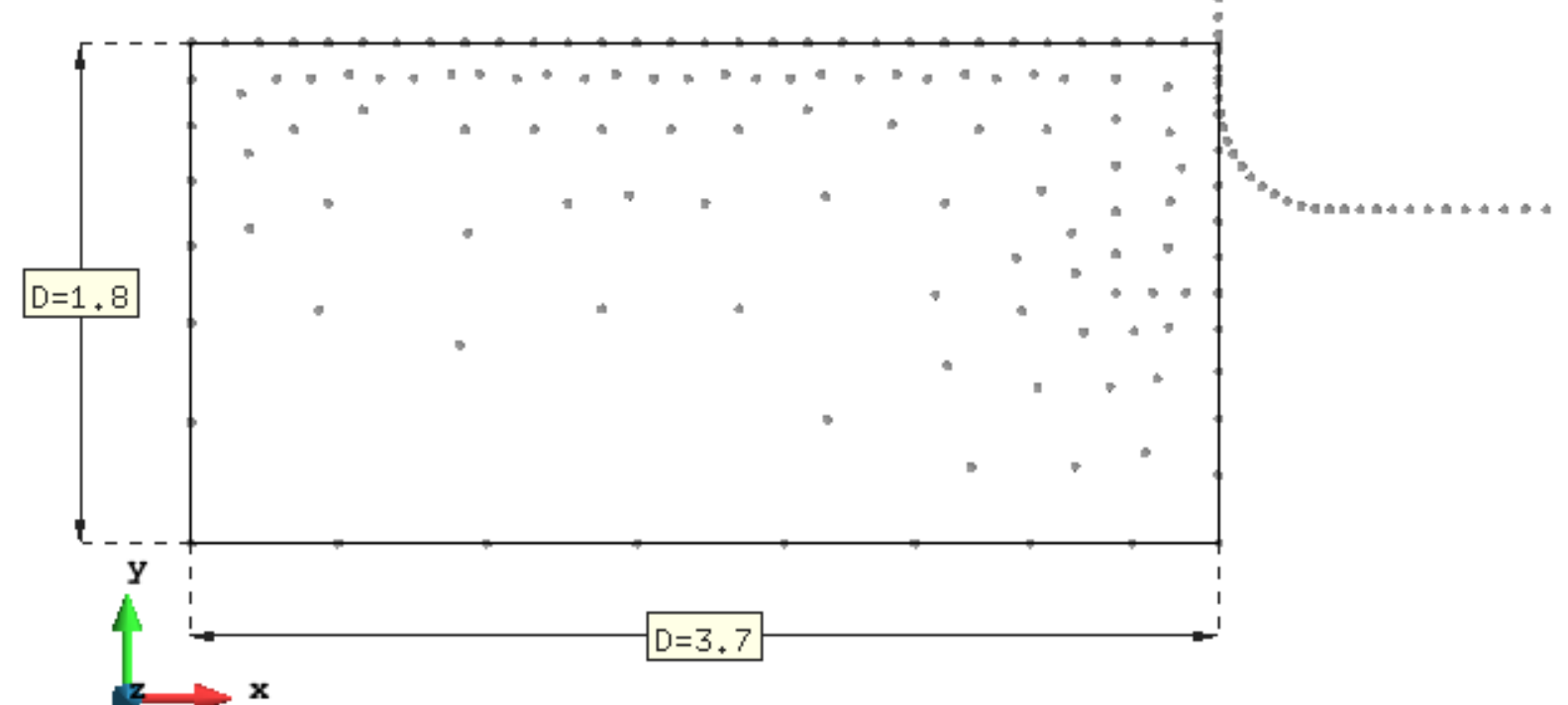

Figure 3. Workpiece dimensions and initial distribution of particles.

\begin{tabular}{|l|l|}
\hline Property & Value \\
\hline Elastic Modulus $(\mathrm{MPa})$ & 210000 \\
\hline Poisson ratio & 0.3 \\
\hline Heat Capacity(N/Km $\left.{ }^{2}\right)$ & $3.5155 \times 10^{6}$ \\
\hline Conductivity(N/sK) & 14 \\
\hline A $(\mathrm{MPa})$ & 860 \\
\hline B $(\mathrm{MPa})$ & 683 \\
\hline C & 0.035 \\
\hline $\mathrm{N}$ & 0.47 \\
\hline $\mathrm{M}$ & 1 \\
\hline$T_{0}(\mathrm{~K})$ & 293.15 \\
\hline$T_{\text {melt }}(\mathrm{K})$ & 1793 \\
\hline
\end{tabular}

Table 4. Materials properties used in the numerical simulations. 
Conductivity and specific heat does not depend on temperature, we consider them constant (see Table 4). The following assumptions are made: First, the tool is supposed to be rigid and friction is neglected. Furthermore, the thermal exchange between the part and the tool are also neglected. The inertia of the part is neglected. A classical penalty method is considered for the contact constraint generated by the action of the rigid tool.

A staggered implicit time integration scheme was used. Time steps were of $1.33 \times 10^{-6} \mathrm{~s}$ which takes 500 steps for a tool to travel $2.22 \mathrm{~mm}$. The assumption that the tool is rigid is reasonable, since the deformation of the tool is negligible compared with the deformation of the workpiece.

Temperature, pressure, effective plastic strain rate and von Mises contours are presented in Figure 4 and Figure 5. Depicted pressure distribution shows the tension and compression zones. It is completed with the von Mises stress shown, which demonstrates that relatively high stresses arise in the primary shear zone and at the tool chip interface. The localization of this zone agrees with simplified models. It is also important to note the presence of residual stresses at and below the produced new surface and in the upper part of the chip, especially near the tool-chip interface where unloading due to curling of the chip occurred. The effective strain rate in the primary and the secondary shear zone is of the order of 18000 and it has its highest value close to the tool tip. Finally, temperature distribution is also shown in the workpiece.

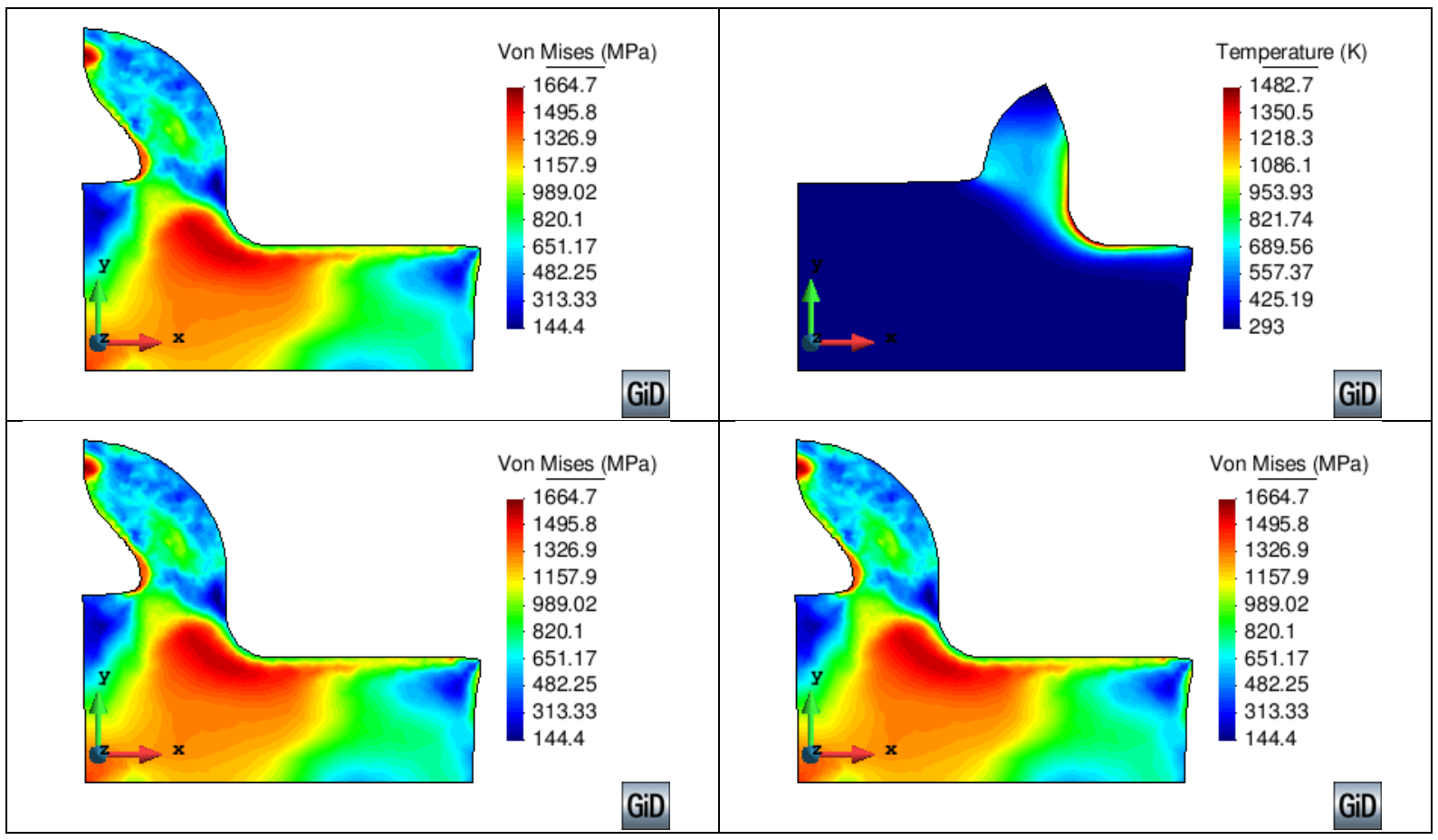

Figure 4. Continuous chip formation using the Johnson-Cook constitutive model: von Mises (MPa) and Temperature (K). 


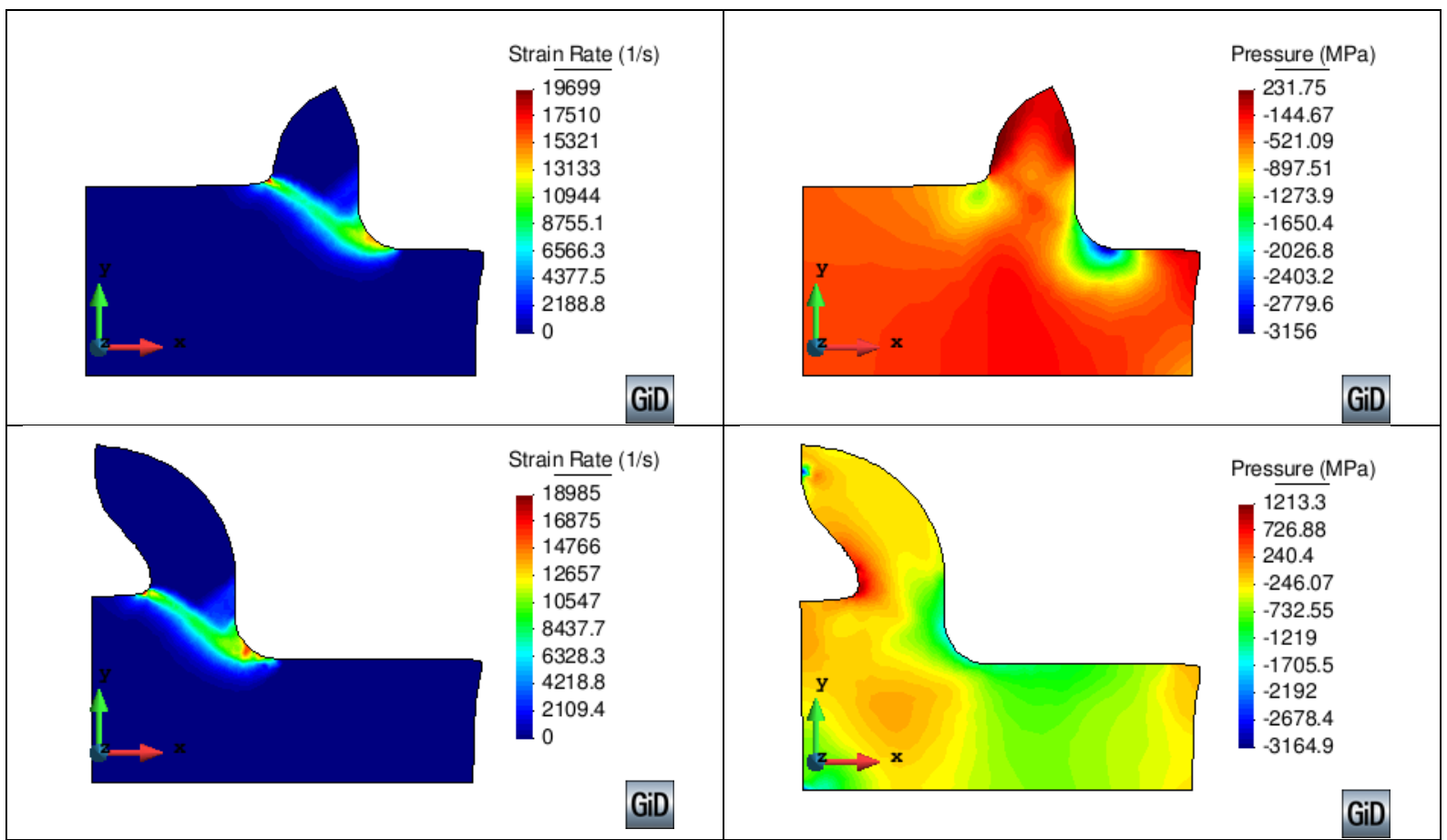

Figure 5. Continuous chip formation using the Johnson-Cook constitutive model: Strain rate (1/s) and Pressure (MPa).

Figure 6 depicts the cutting and feed forces applied on the tool, which are obtained from the simulation. The cutting and feed force reach a steady state as expected in the numerical simulation of continuous chip formation.

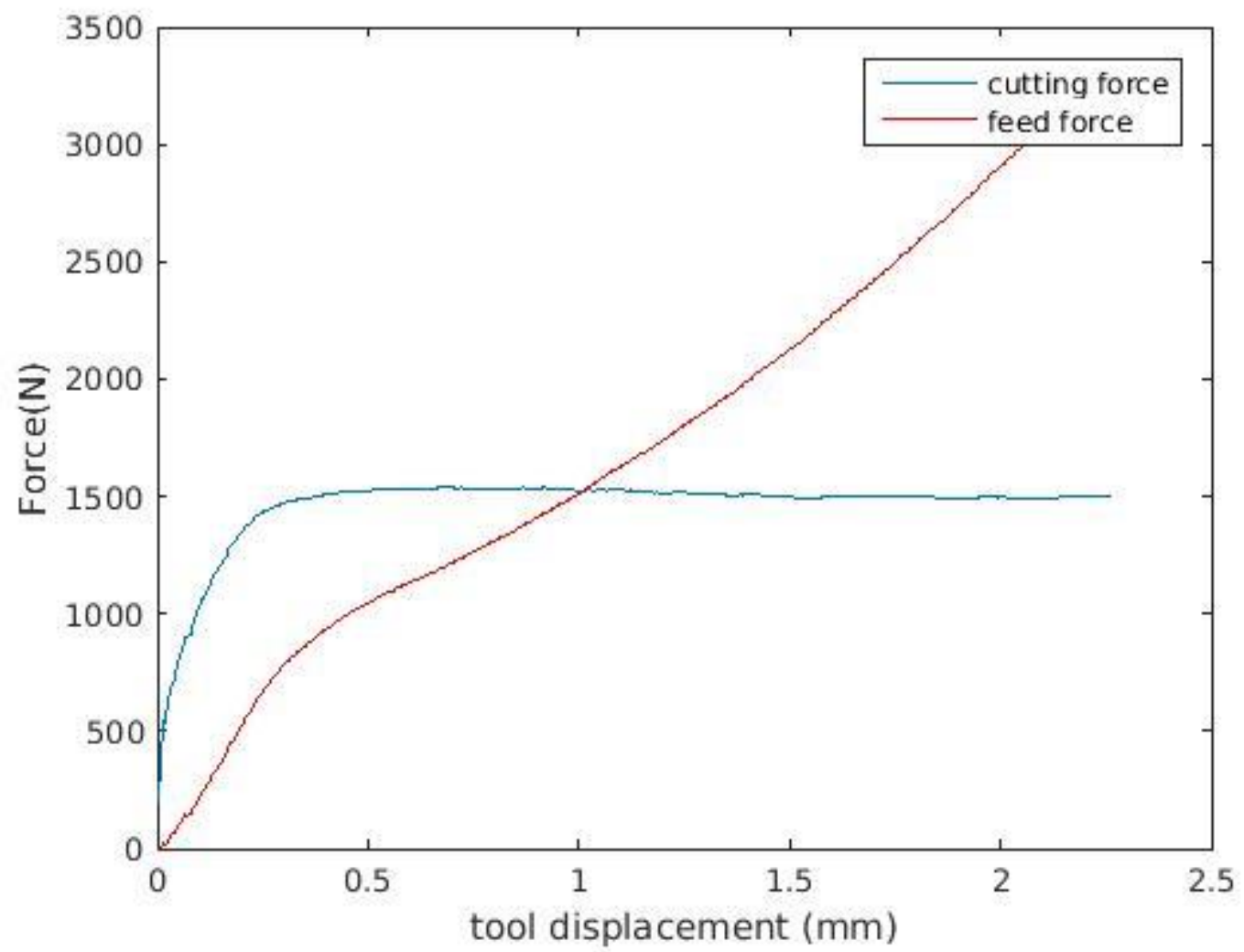

Figure 6. Cutting and thrust force vs. stool displacement. 


\subsection{Orthogonal cutting test: 3D approach}

The material properties for the workpiece, the cutting conditions are the same used in example 5.1. Also, the displacement in the $\mathrm{x}$-direction of front and back faces of the workpiece is restricted to reproduce a plain strain conditions (see example 5.1). The initial finite element meshes for the workpiece and the tool are shown in Figure 7. The workpiece is modeled with 569 particles and the tool with 1592 triangle elements. For saving calculation time and memory space, insertion and removal of particles is used during the simulation. As a consequence, the finer and smaller elements are used in the contact area between the workpiece and the tool because very large gradients in strains and temperature will occurs in this zone. The coarser and the larger elements are allocated in the area of the workpiece not affected by the cutting processes.

Figure 8 shows a three dimensional view of the chip geometry predicted when the tool advances from 0 to $2.22 \mathrm{~mm}$. Temperature distribution is presented in Figure 9. Because temperature increase is primary induced by plastic deformation, the more severe a deformation zone is, the greater the temperature increase.

The cutting force for the 2D and 3D simulations are show in Figure 10. The results for the $2 \mathrm{D}$ and $3 \mathrm{D}$ are qualitatively the same, the small different between both is due to the larger distance between particles used in the 3D numerical simulations.
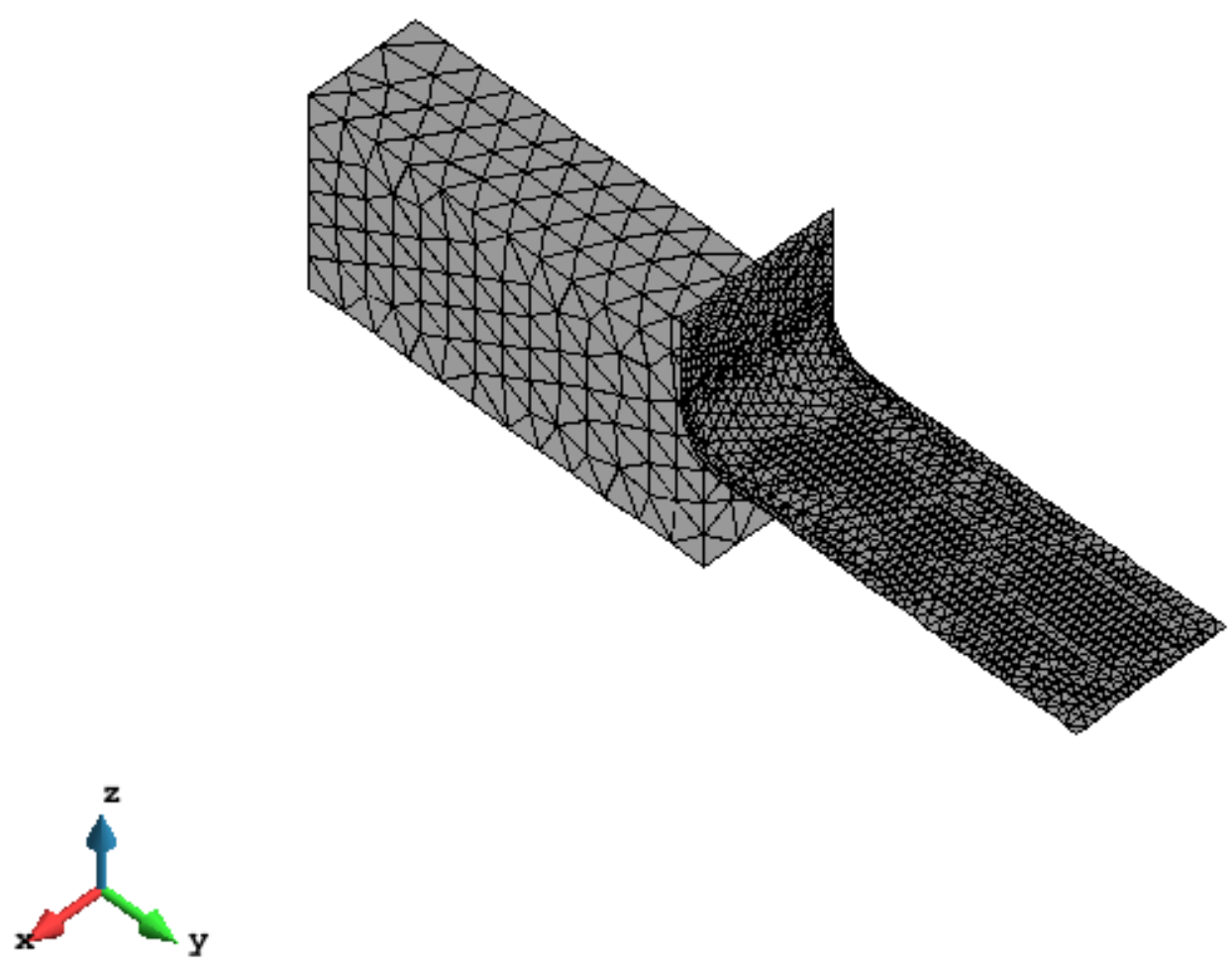

Figure 7. Workpiece and cutting tool discretization. 


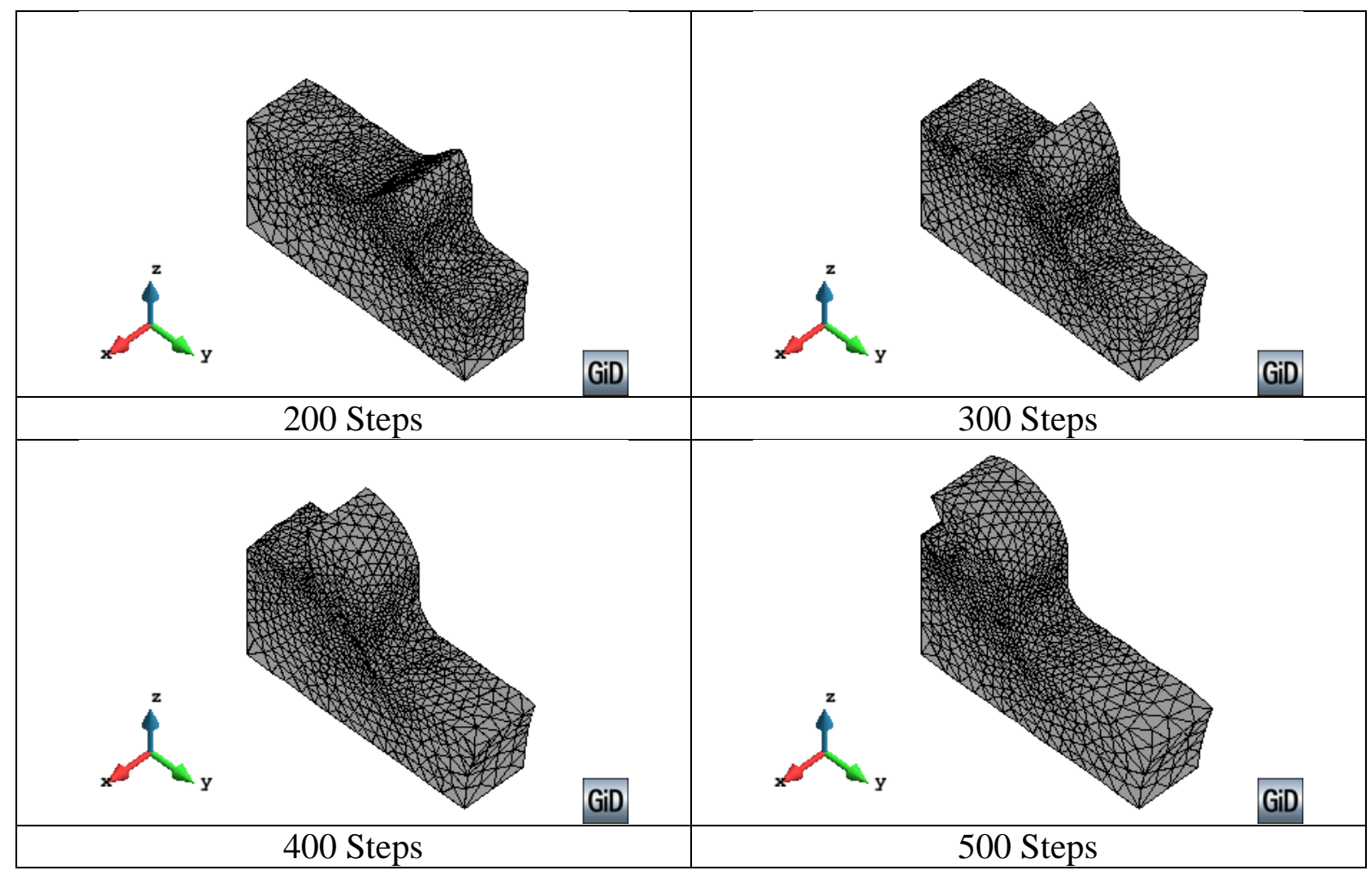

Figure 8. Chip geometries at different time steps during orthogonal cutting.

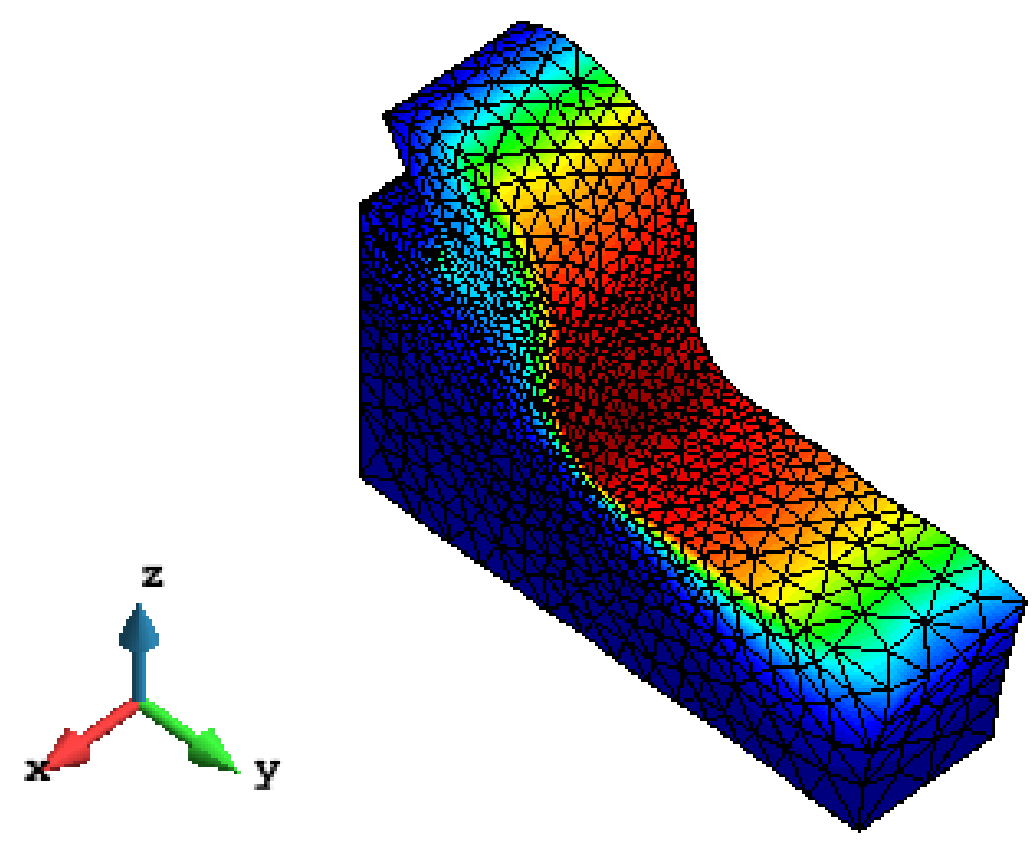

Temperature (K)

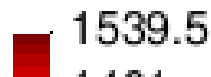

1401

1262.5

1124

985.56

847.07

708.59

570.11

431.63

293.15

Figure 9. Temperature distribution $(K)$ in the workpiece an the chip (the cutting tool is $2.22 \mathrm{~mm}$ ) 


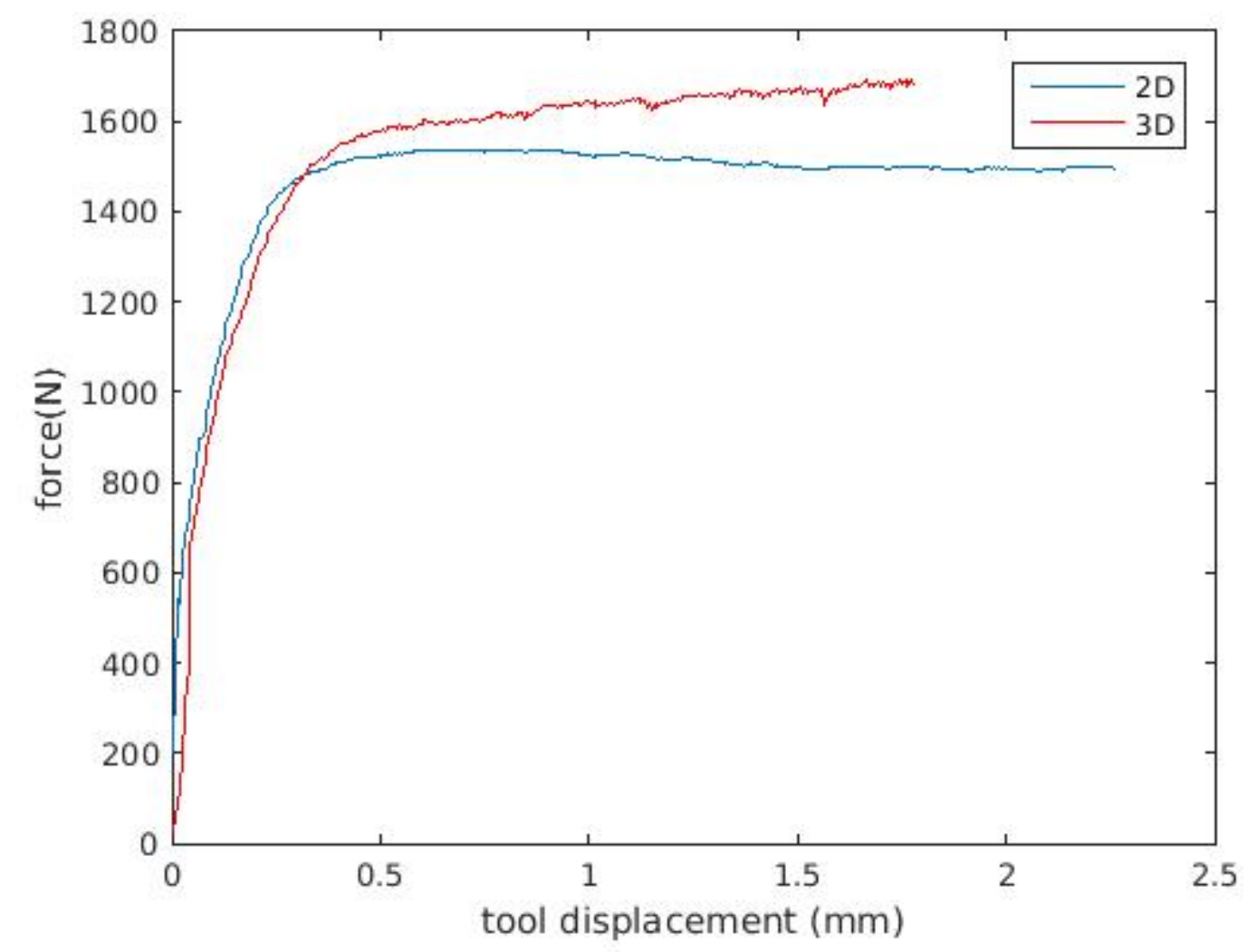

Figure 10. Force displacement curve for orthogonal cutting for $2 \mathrm{D}$ and $3 \mathrm{D}$ case.

\section{CONCLUSIONS}

A Lagrangian formulation for analysis of metal cutting processes involving thermally coupled interactions between deformable continua is presented. The governing equations for the generalized continuum are discretized using elements with equal linear interpolation for the displacement and the temperature. The merits of the formulation in terms of its general applicability have been demonstrated in the solution two representative numerical simulations of orthogonal cutting using the PFEM both in 2D and 3D. Results are promising for future numerical simulations of metal cutting processes

\section{REFERENCES}

[1] T. D. Marusich and M. Ortiz, "Modelling and simulation of high-speed machining," International Journal for Numerical Methods in Engineering, vol. 38, pp. 3675-3694, 1995.

[2] G. S. Sekhon and J. L. Chenot, "Numerical simulation of continuous chip formation during non-steady orthogonal cutting simulation," Engineering Computations, vol. 10, 1993.

[3] L. Illoul and P. Lorong, "On some aspects of the CNEM implementation in 3D in order to simulate high speed machining or shearing," Computer and Structures, vol. 89, pp. 940-958, 2011.

[4] F. Chinesta, P. Lorong, D. Ryckelynck, M. Martinez, E. Cueto, M. Doblaré, G. Coffignal, M. Touratier, and J. Yvonnet, "Thermomechanical cutting model discretiation: eulerian or lagrangian, mesh or meshless?," International Journal of Forming Processes, vol. 7, pp. 83-97, 2004. 
[5] F. Greco, L. Filice, C. Peco, and M. Arroyo, "A stabilized formulation with maximum entropy meshfree approximants for viscoplastic flow simulation in metal forming," INTERNATIONAL JOURNAL OF MATERIAL FORMING, vol. 8, pp. 341-353, 2015/07/01 2015.

[6] A. Raczy, M. Elmadagli, W. J. Altenhof, and A. T. Alpas, "An eulerian finite-element model for determination of deformation state of a copper subjected to orthogonal cutting," Metalurgical and Materials Transactions A, vol. 35, pp. 2393-2400, 2004.

[7] L. Olovsson, L. Nilsson, and K. Simonsson, "An ALE formulation for the solution of two-dimensional metal cutting problems," Computers \& Structures, vol. 72, pp. 497507, 1999.

[8] R. Rakotomalala, P. Joyot, and M. Touratier, "Arbitrary Lagrangian-Eulerian thermomechanical finite-element model of material cutting," Communications in Numerical Methods in Engineering, vol. 9, pp. 975-987, 1993.

[9] M. Movahhedy, M. S. Gadala, and Y. Altintas, "Simulation of the orthogonal metal cutting process using an arbitrary Lagrangian-Eulerian finite-element method," Journal of Materials Processing Technology, vol. 103, pp. 267-275, 2000.

[10] E. Uhlmann, R. Gerstenberger, M. Graf von der Schulenburg, J. Kurnert, and A. Mattes, "The Finite-Pointset-Method for the Meshfree Numerical Simulation of Chip Formation," presented at the 12 Cirp Conference on Modelling of Machining Operations, San Sebastian, Spain, 2009.

[11] E. Uhlmann, R. Gerstenberger, and J. Kuhnert, "Cutting Simulation with the Meshfree Finite Pointset Method," Procedia CIRP, vol. 8, pp. 391-396, 2013.

[12] C. R. Dohrmann and P. B. Bochev, "A stabilized finite element method for the Stokes problem based on polynomial pressure projections," International Journal for Numerical Methods in Fluids, vol. 46, pp. 183-201, 2004.

[13] J. M. Rodriguez, J. M. Carbonell, J. C. Cante, and J. Oliver, "The particle finite element method (PFEM) in thermo-mechanical problems," International Journal for Numerical Methods in Engineering, pp. n/a-n/a, 2015.

[14] H. Edelsbrunner, E. P. M, \#252, and cke, "Three-dimensional alpha shapes," ACM Trans. Graph., vol. 13, pp. 43-72, 1994.

[15] J. M. Rodríguez, J. C. Cante, and X. Oliver, "On the numerical modelling of machining processes via the Particle finite Element method (PFEM)," vol. 156, ed. Barcelona: CIMNE, 2015, p. 186.

[16] J. C. Simo, "A framework for finite strain elastoplasticity based on maximum plastic dissipation and the multiplicative decomposition: part I. continuum formulation," Computer Methods in Applied Mechanics and Engineering archive, vol. 666, pp. 199219, 1988.

[17] J. C. Simo, "A framework for finite strain elastoplasticity based on maximum plastic dissipation and the multiplicative decomposition. Part II: Computational aspects," Computer Methods in Applied Mechanics and Engineering, vol. 68, pp. 1-31, 1988.

[18] J. C. Simo and C. Miehe, "Associative coupled thermoplasticity at finite strains: Formulation, numerical analysis and implementation," Computer Methods in Applied Mechanics and Engineering, vol. 98 pp. 41-104, 1992.

[19] G. H. Johnson and W. H. Cook, "A constitutive model and data for metals subjected to large strains high strain rates and high temperatures," Proceedings of the 7th symposium on ballistics, 1983. 